Alberta-Thy-07-99

\title{
Statistical Mechanics on Axially-symmetric Space-times with the Killing Horizon and Entropy of Rotating Black Holes in Induced Gravity
}

\author{
V.P. Frolov ${ }^{* 1}$ and D.V. Fursaev ${ }^{\dagger 2}$ \\ 1 Theoretical Physics Institute, Department of Physics, University of Alberta \\ Edmonton, Canada T6G 2J1 and \\ ${ }^{2}$ Joint Institute for Nuclear Research, Bogoliubov Laboratory of Theoretical Physics, \\ 141980 Dubna, Russia
}

\begin{abstract}
We develop a method for computing the free-energy of a canonical ensemble of quantum fields near the horizon of a rotating black hole. We show that the density of energy levels of a quantum field on a stationary background can be related to the density of levels of the same field on a fiducial static space-time. The effect of the rotation appears in the additional interaction of the "static" field with a fiducial abelian gauge-potential. The fiducial static space-time and the gauge potential are universal, i.e., they are determined by the geometry of the given physical spacetime and do not depend on the spin of the field. The reduction of the stationary axially symmetric problem to the static one leads to a considerable simplification in the study of statistical mechanics and we use it to draw a number of conclusions. First, we prove that divergences of the entropy of scalar and spinor fields at the horizon in the presence of rotation have the same form as in the static case and can be removed by renormalization of the bare black hole entropy. Second, we demonstrate that statistical-mechanical representation of the Bekenstein-Hawking entropy of a black hole in induced gravity is universal and does not depend on the rotation.
\end{abstract}

PACS number(s): 04.60.+n, 12.25.+e, 97.60.Lf, 11.10.Gh

*e-mail: frolov@phys.ualberta.ca

†e-mail: fursaev@thsun1.jinr.ru 


\section{Introduction}

There are different approaches to the explanation of the Bekenstein-Hawking entropy $S^{B H}$ of black holes. One of them is to relate $S^{B H}$ to the statistical-mechanical entropy $S^{S M}$ of the thermal atmosphere of quantum fields near the black hole horizon [1]-[0]. $S^{S M}$ can be naturally interpreted as the entropy of entanglement [3]-[5] which arises as the result of quantum correlations on the horizon. For a review and recent development of these ideas see [6] and references therein.

Computation of $S^{S M}$ is a delicate procedure because the density of energy levels $d n / d \omega$ of single-particle excitations is divergent near the horizon. On static space-times the wave equation for a mode with the certain energy $\omega$ is similar to a relativistic Schroedinger equation. In this case, one can define a single-particle Hamiltonian $H$ as the "square root" of a Laplace operator acting on a space whose 3-geometry is approximated by the hyperbolic geometry. It enables one to use rigorous methods to investigate the spectrum $\omega$ of $H$ and find its density $d n / d \omega[0]-[9]$. However, this approach cannot be applied directly to fields near a rotating black hole. The difficulty is that in the latter case the wave equation which determines the spectrum of energies contains terms which are both quadratic and linear in $\omega$. As a result, one cannot define the single-particle Hamiltonian by taking naively the "square root" prescription.

There was no rigorous and universal method how to avoid this difficulty in computing $S^{S M}$ for rotating black holes. For this reason, many authors used WKB approximation [10] [15] or alternative [16] approaches. These approaches were always based on approximations and, hence, require a justification. The calculations of the entropy were also done by Mann and Solodukhin, who were using the Euclidean formalism [17- 18. In the recent paper [15] Jing and Yan demonstrated an agreement of the WKB calculations of the black hole entropy for rotating black holes with the Euclidean results.

The aim of this work is two-fold. First, we suggest a general method for computing the spectrum and doing statistical-mechanical computations in the case of rotating black holes. Second, we draw with its help a number of consequences concerning the entropy $S^{S M}$. The proposed method uses the covariant Schwinger-DeWitt technique and it can be applied to fields of different spins.

The terms in the wave equation which are linear in $\omega$ appear due to the non-vanishing component $g_{t \varphi}$ of the background metric. Our idea is to include these terms in the definition of a fiducial single particle Hamiltonian $H(\omega)$ which depends on the energy $\omega$ as on additional parameter. The operator $H(\omega)$ can be interpreted as the Hamiltonian of a particle moving on a fiducial background and interacting with an external fiducial gauge potential with the only non-vanishing component $A_{\varphi} \sim g_{t \varphi}$. In some regard the appearance of the potential is analogous to the origin of the gauge field from the component $g_{5 \mu}$ of the metric in Kaluza-Klein compactifications.

Thus, our method is to reduce the problem of computations on the stationary back- 
ground to computations on a fiducial static space-time with external gauge field, i.e., to the problem which is already solved. As we will see, the form of the fiducial background and gauge field is determined by the geometry of the physical space-time only and is the same for the fields of different spins. In this sense, the method is universal. Moreover, the method can be applied to black holes in arbitrary dimensions.

The paper is organized as follows. In Section 2 we introduce the fiducial background and demonstrate our method for scalar and spinor fields. In Section 3 we use the obtained results to derive the one-loop divergences of the entropy $S^{S M}$ for a rotating black hole in a most complete form. We show that this form is the same as in the case of static space-time and, hence, the known renormalization procedure [19]-22] can be applied to remove the divergences. In Section 4 we discuss black hole entropy in models of induced gravity 23], 24]. We use the obtained results to prove that the statistical-mechanical form of the Bekenstein-Hawking entropy $S^{B H}$ for static and rotating black holes in this theory is the same. This fact may be considered as another evidence that the mechanism of generating the black hole entropy [25] is universal. Our concluding remarks are presented in Section 5. In Appendix we demonstrate that geometrical characteristics of the physical and fiducial backgrounds at the horizon coincide. We use this property in the main text in the paper.

\section{Statistical mechanics in a space-time of a rotating black hole}

\subsection{Stationary axisymmetric space-times}

We begin with the formulation of statistical-mechanics of scalar and spinor fields on a stationary axially symmetric space-times with a Killing horizon. Consider a $D$-dimensional space-time $\mathcal{M}$ with two commuting Killing vector fields $\partial_{t}$ and $\partial_{\varphi}$. We assume that the vector $\partial_{t}$ is time-like at asymptotic infinity, and is normalized at infinity by the condition $\partial_{t} \cdot \partial_{t}=-1$. The other Killing vector $\partial_{\varphi}$ corresponds to the symmetry of space with respect to rotation. It commutes with $\partial_{t}$ and has closed integral curves. The field $\partial_{\varphi}$ is nonzero everywhere in the exterior region and at the horizon, except on the rotation axis. We also assume that at the rotation axis space-time is locally flat (i.e., there is no conical singularities). The vector fields $\partial_{t}$ and $\partial_{\varphi}$ possessing the properties described above are uniquely defined in a axially symmetric asymptotically flat space-time.

In such a space-time one can introduce coordinates $t, \varphi, x^{k}(k=2, \ldots, D-2)$ in which the metric takes the form

$$
d s^{2}=g_{t t} d t^{2}+2 g_{t \varphi} d t d \varphi+g_{\varphi \varphi} d \varphi^{2}+g_{i k} d x^{i} d x^{k}
$$

${ }^{1}$ Strictly speaking, this is true for vacuum $4 D$ space-times. In a more general case, when matter or fields are present, a so-called circularity condition must be satisfied. See, e.g. [26]. 
Here $0 \leq \varphi \leq 2 \pi$, and the components of the metric depend on coordinates $x^{k}$ only.

We assume that a stationary asymptotically flat space-time $\mathcal{M}$ contains a rotating black hole and is a solution of Einstein equations with matter satisfying suitable hyperbolic equations. In this case, the event horizon $H$ coincides with the Killing horizon [27]. The latter is defined as a null surface, $H$, to which a Killing vector $\xi$ is normal. In the stationary axisymmetric space-time the Killing vector $\xi$ can be written as

$$
\xi=\partial_{t}+\Omega_{H} \partial_{\varphi}
$$

Here $\Omega_{H}$ is the angular velocity of the black hole which is constant at the horizon. The position of the horizon $H$ is determined by the equation

$$
\left(g_{t \varphi}\right)^{2}-g_{t t} g_{\varphi \varphi}=0
$$

while the angular velocity $\Omega_{H}$ is

$$
\Omega_{H}=-\left.\frac{g_{t \varphi}}{g_{\varphi \varphi}}\right|_{H}
$$

For our purpose, it is convenient to rewrite metric (2.1) in the coordinates which are rigidly co-rotating with the black hole. Let

$$
\tilde{\varphi}=\varphi-\Omega_{H} t
$$

then metric (2.1) takes the form

$$
d s^{2}=-N^{2} d t^{2}+g_{\varphi \varphi}\left(d \tilde{\varphi}+\tilde{N}_{\varphi} d t\right)^{2}+g_{i k} d x^{i} d x^{k}
$$

Here,

$$
N^{2} \equiv-\frac{1}{g^{t t}}=\frac{\left(g_{t \varphi}\right)^{2}-g_{t t} g_{\varphi \varphi}}{g_{\varphi \varphi}} \quad, \quad \tilde{N}^{\varphi}=N^{\varphi}+\Omega_{H} \quad, \quad N^{\varphi} \equiv \frac{g_{t \varphi}}{g_{\varphi \varphi}} .
$$

It is evident that

$$
\left.\tilde{N}^{\varphi}\right|_{H}=0
$$

From equation (2.3) it follows that $N^{2}=0$ on the horizon. (At the axis of symmetry $N^{2}$ can be defined by continuity.) By using the condition of the regularity of the metric on the horizon it is possible to show that the ratio $\tilde{N}^{\varphi} / N^{2}$ is not singular on $H$.

One can also rewrite the line element (2.6) in the form which will be especially useful for our purposes

$$
d s^{2}=-B(d t-W d \tilde{\varphi})^{2}+C d \tilde{\varphi}^{2}+g_{i k} d x^{i} d x^{k}=-B(d t-W d \tilde{\varphi})^{2}+d l^{2} \quad .
$$

Here

$$
B=-\xi^{2}=N^{2}\left(1-g_{\varphi \varphi} \frac{\left(\tilde{N}^{\varphi}\right)^{2}}{N^{2}}\right)
$$




$$
\begin{gathered}
\frac{1}{C}=\frac{1}{g_{\varphi \varphi}}\left(1-g_{\varphi \varphi} \frac{\left(\tilde{N}^{\varphi}\right)^{2}}{N^{2}}\right), \\
W=C \frac{\tilde{N}^{\varphi}}{N^{2}} .
\end{gathered}
$$

Consider a Killing observer, that is the observer which has the velocity $u^{\mu} \sim \xi^{\mu}$. Let a point $p$ lying on the worldline of this observer has coordinates $x^{\mu}=\left(t, \tilde{\varphi}, x^{i}\right)$. The condition that another event $y^{\mu}=\left(t+d t, \tilde{\varphi}+d \tilde{\varphi}, x^{i}\right)$ in its vicinity is simultaneous with $p$, that is it lies in the plane orthogonal to $\xi$, implies $d t=W d \tilde{\varphi}$. The spatial distance between these two events is $d l$.

In the general case, the horizon of a rotating black hole is surrounded by the region called the ergosphere. Inside the ergosphere $\xi$ is time-like, while the vector $\partial_{t}$ is space-like.

\subsection{Scalar fields}

Let us now investigate the properties of the spectrum of single-particle excitations in a space-time of a stationary rotating black hole. We begin with the free scalar field which is described by the Klein-Gordon equation

$$
\begin{gathered}
\left(-\nabla^{\mu} \nabla_{\mu}+V\right) \phi=0, \\
V=\xi R+m^{2} .
\end{gathered}
$$

In accordance with the assumed symmetry, we can write a solution of this equation by using decomposition into modes

$$
\begin{gathered}
\phi_{\omega, l}(t, \varphi, \mathbf{x})=e^{-i\left(\omega+\Omega_{H} l\right) t+i l \varphi} \phi_{\omega, l}(\mathbf{x}) \\
i \xi \phi_{\omega, l}(t, \varphi, \mathbf{x})=\omega \phi_{\omega, l}(t, \varphi, \mathbf{x}) \\
-i \partial_{\varphi} \phi_{\omega, l}(t, \varphi, \mathbf{x})=l \phi_{\omega, l}(t, \varphi, \mathbf{x})
\end{gathered}
$$

where $\mathbf{x}$ are the rest coordinates of $\mathcal{M}$. The corresponding single-particle excitation of a scalar field has energy $\omega$ (defined with respect to the Killing vector $\xi$ ) and the integer angular momentum $l$. In the co-rotating coordinates the wave functions (2.15) take the familiar form

$$
\phi_{\omega, l}\left(t, \tilde{\varphi}+\Omega_{H} t, \mathbf{x}\right)=e^{-i \omega t+i l \tilde{\varphi}} \phi_{\omega, l}(\mathbf{x})
$$

Equation for the spectrum $\omega$ follows from (2.1) after substitution function (2.15). One easily finds the relation

$$
\left[\frac{1}{N^{2}}\left(\omega+l \tilde{N}^{\varphi}\right)^{2}-\frac{1}{g_{\varphi \varphi}} l^{2}-\Delta_{\mathbf{x}}-V\right] \phi_{\omega, l}(\mathbf{x})=0
$$

where

$$
\Delta_{\mathbf{x}} \equiv-\frac{1}{\sqrt{-g}} \partial_{i}\left[\sqrt{-g} g^{i k} \partial_{k}\right]
$$


The presence of linear in $\omega$ terms in this equation makes it difficult to use the standard methods for obtaining the density of energy levels for this operator. We shall demonstrate now that this problem can be reduced to the problem in a static space-time.

Proposition: The spectrum of single-particle excitations for the wave operator 2.13)(2.14) in space-time (2.1) is uniquely defined by the spectrum of single-particle excitations for the wave operator

$$
\left[-\tilde{g}^{\mu \nu}\left(\tilde{\nabla}_{\mu}-i \lambda A_{\mu}\right)\left(\tilde{\nabla}_{\nu}-i \lambda A_{\nu}\right)+V\right] \phi^{(\lambda)}=0
$$

on a static background $\tilde{\mathcal{M}}$ with the metric

$$
d \tilde{s}^{2}=\tilde{g}_{\mu \nu} d x^{\mu} d x^{\nu}=-B d t^{2}+C d \varphi^{2}+g_{i k} d x^{i} d x^{k}
$$

and the abelian gauge field

$$
A=W d \varphi
$$

provided that metric coefficients $B$ and $C$, and the field potential $W$ are given by relations (2.10), (2.11), and (2.19), respectively.

Equation (2.21) contains a real non-negative parameter $\lambda$ which can be interpreted as the electric charge of the field $\phi^{(\lambda)}$. The correspondence between the spectra means that a single-particle excitation with the energy $\omega$ for wave operator (2.13)-(2.14) in spacetime (2.1) is uniquely related to a single-particle excitation for operator (2.21) taken at $\lambda=\omega$ and having the same energy $\omega$. For a static space-time $B=N^{2}, C=g_{\varphi \varphi}, W=0$ and two problems are equivalent. In general case, the geometry of space $\tilde{\mathcal{M}}$ differs from the geometry of physical space-time $\mathcal{M}$. To emphasize this difference we call $A$ and $\tilde{\mathcal{M}}$ the fiducial gauge field and the fiducial background, respectively. The reduction of our problem to the static one on $\tilde{\mathcal{M}}$ makes it possible a considerable simplification in the computations which we use in a moment.

We now prove the above proposition. Let us first rewrite Eq. (2.19) in the following equivalent form

$$
\left[\omega^{2}-B\left(\Delta_{\mathbf{x}}+\frac{1}{C}(l-\omega W)^{2}+V\right)\right] \phi_{\omega, l}(\mathbf{x})=0
$$

where $B, C$, and $W$ are given by relations (2.10)-(2.12). Equation (2.24) is the Fourier transform of the differential equation

$$
\begin{gathered}
{\left[\omega^{2}-B\left(\Delta_{\mathbf{x}}-\frac{1}{C}\left(\partial_{\varphi}-i \omega W\right)^{2}+V\right)\right] \phi_{\omega}(\varphi, \mathbf{x})=0} \\
\phi_{\omega}(\varphi, \mathbf{x})=\sum_{l} e^{i l \varphi} \phi_{\omega, l}(\mathbf{x})
\end{gathered}
$$


Let us introduce the second order differential operator on a $D$-1-dimensional space

$$
H^{2}(\lambda)=B\left(\Delta_{\mathbf{x}}-\frac{1}{C}\left(\partial_{\varphi}-i \lambda W\right)^{2}+V\right)
$$

where $\lambda$ is a real parameter. Let $\phi_{\omega}^{(\lambda)}$ be eigen-functions of $H^{2}(\lambda)$

$$
H^{2}(\lambda) \phi_{\omega}^{(\lambda)}=\omega^{2} \phi_{\omega}^{(\lambda)}
$$

Obviously, the eigen-functions $\phi_{\omega}^{(\lambda)}$ enable one to solve the eigen-problem (2.25) because

$$
\phi_{\omega}(\varphi, \mathbf{x})=\phi_{\omega}^{(\omega)}(\varphi, \mathbf{x})
$$

Let us define now the field

$$
\phi^{(\lambda)}(t, \varphi, \mathbf{x})=\sum_{\omega} e^{-i \omega t} \phi_{\omega}^{(\lambda)}(\varphi, \mathbf{x})
$$

Then it is not difficult to see that the eigen-value problem (2.28) is equivalent to the Klein-Gordon equation (2.21) in the space-time (2.22) with the Abelian field (2.23). To come to this conclusion one has to use the identity

$$
B C=N^{2} g_{\varphi \varphi}
$$

which is the consequence of (2.11) and (2.10). By using (2.28) and (2.29) one also obtains the density of the energy-levels $\omega$ in $(2.26), d n(\omega) / d \omega$, as the density of the energy levels $d n^{(\lambda)}(\omega) / d \omega$ of the operator $H^{2}(\lambda)$ at $\lambda=\omega$

$$
\frac{d n(\omega)}{d \omega}=\left.\frac{d n^{(\lambda)}(\omega)}{d \omega}\right|_{\lambda=\omega} .
$$

This equation completes the proof of the proposition.

Equation (2.32) is important because the density of the energy-levels plays a crucial role in the definition of the free energy of the system

$$
F[\beta]=\eta \beta^{-1} \int d \omega \frac{d n(\omega)}{d \omega} \ln \left(1-\eta e^{-\beta \omega}\right)
$$

where $\beta$ is the inverse temperature and $\eta=+1$ for bosons and $\eta=-1$ for fermions. Finding the quantity $d n^{(\lambda)}(\omega) / d \omega$ enables one to determine all statistical-mechanical characteristics of the canonical ensemble on the axially-symmetric background, including the entropy.

At this point several remarks are in order. As follows from (2.27), the operator $H^{2}(\lambda)$ is positive when $B=-\xi^{2}>0$, that is in the region of the black-hole exterior lying between the horizon and the null 'cylinder', a surface where the co-rotation velocity reaches the velocity of light. Outside of this region Eq. (2.25) may not have solutions for real values 
of energies $\omega$. This property is the manifestation of the superradiance phenomenon in the gravitational field of a rotating black hole. In the presence of superradiance, there does not exist a stationary regular in the black hole exterior quantum state. In order to escape the problem connected with the superradiant modes it is possible to introduce a mirror-like boundary surrounding the black hole. One can define a canonical ensemble for the quantum field inside such a boundary, provided it is chosen to be close enough to the black hole (inside the null 'cylinder'). In what follows we assume that such a boundary does exist. Note, however, that we shall be interested in the entropy which is determined by the region in the vicinity of the horizon, and, hence, the leading divergent contribution to the entropy does not depend on the outer boundary.

Also it should be emphasized once again why we define the energy of the canonical ensemble with respect to the Killing field $\xi$ rather than vector $\partial_{t}$. The reason is that our final goal is to compute the entanglement entropy $S^{S M}$ of fields. The origin of the entanglement entropy is closely related to the presence of the horizon and the structure of the Killing field $\xi$. In case of black hole the entanglement entropy has a thermal nature and it can be defined as the entropy of the thermal atmosphere around a black hole. More formally, it can be shown that the entanglement density matrix for a rotating black hole is $\hat{\rho} \sim \exp \left(-\hat{H} / T_{H}\right)$ where $\hat{H}$ is the generator of canonical transformations along the Killing field $\xi$ and $T_{H}$ is the Hawking temperature, see, e.g., [28]. In this regard, our approach is different from the approach [13] where the energy is determined with respect to the vector $\partial_{t}$. In the latter case one always has to deal with the contribution of the superradiant modes which appear because $\partial_{t}$ is space-like near the horizon. Thus, although the vector $\partial_{t}$ can be used to define the energy at spatial infinity, it is not related to the notion of entanglement entropy.

As follows from (2.25), $H^{2}(\lambda)$ is Hermitean operator with respect to the inner product

$$
\left(\phi_{1}, \phi_{2}\right)=\int d \varphi d^{D-2} \mathbf{x} \sqrt{-g B^{-2}} \phi_{1}^{*}(\varphi, \mathbf{x}) \phi_{2}(\varphi, \mathbf{x})
$$

Following the procedure elaborated in the case of static space-times [9] it is convenient to introduce another representation of $H^{2}(\lambda)$

$$
\begin{gathered}
\bar{H}^{2}(\lambda)=e^{-\frac{D-2}{2} \sigma} H^{2}(\lambda) e^{\frac{D-2}{2} \sigma}, \\
e^{-2 \sigma}=B
\end{gathered}
$$

In the new representation

$$
\begin{gathered}
\bar{H}^{2}(\lambda)=-\bar{g}^{a b}\left(\bar{\nabla}_{a}-i \lambda A_{a}\right)\left(\bar{\nabla}_{b}-i \lambda A_{b}\right)+\bar{V} \\
\bar{V}=B V+\frac{D-2}{2}\left(\frac{D-2}{2}(\bar{\nabla} \sigma)^{2}-\bar{\nabla}^{2} \sigma\right)= \\
B\left[V+\frac{D-2}{2}\left(\nabla^{\mu} w_{\mu}-\frac{D-2}{2} w^{\mu} w_{\mu}\right)\right] .
\end{gathered}
$$


The indexes $a, b$ in Eq. (2.37) run from 1 to $D-1$ and connections $\bar{\nabla}_{a}$ are determined for the metric

$$
d \bar{l}^{2}=\bar{g}_{a b} d x^{a} d x^{b}=\frac{1}{B}\left(C d \varphi^{2}+g_{i j} d x^{i} d x^{j}\right) .
$$

As earlier the fiducial vector $A_{a}$ is defined by Eq. (2.23). Finally, the vector $w_{\mu}$ in (2.38) is

$$
w_{\mu}=\frac{1}{2} \nabla_{\mu} \ln B
$$

This vector can be interpreted as an acceleration of a static observer in the fiducial spacetime $\tilde{\mathcal{M}}$.

The operator $\bar{H}^{2}(\lambda)$ is Hermitean with respect to the standard inner product

$$
\left(\phi_{1}, \phi_{2}\right)=\int d \varphi d^{D-2} \mathbf{x} \sqrt{\bar{g}} \phi_{1}^{*}(\varphi, \mathbf{x}) \phi_{2}(\varphi, \mathbf{x})
$$

In the static space-time $B=-g_{t t}$ and $w_{\mu}$ coincides with the acceleration in the physical space-time. In four dimensional static space-time Eq. (3.4) reproduces the result of [6], [9].

The operator $\bar{H}(\lambda)$ is the Hamiltonian of a relativistic particle which propagates in the space $\overline{\mathcal{B}}$ with the metric $\bar{g}_{a b}$. The effect of the rotation of the initial space-time is encoded in the properties of the background metric and in the presence of an additional gauge field $A_{a}$. In the static limit the operator $\bar{H}(\lambda)$ does not depend on $\lambda$ and coincides with the single-particle Hamiltonian considered in Refs. [6], [9].

\subsection{Spinor fields}

We now show that the reduction of the stationary problem to the problem on a fiducial static space is universal and also possible for fields with non-zero spins. As an important example we consider spinor fields $\psi$ obeying the Dirac equation

$$
\left(\gamma^{\mu} \nabla_{\mu}+m\right) \psi=0
$$

The spinor derivatives are $\nabla_{\mu}=\partial_{\mu}+\Gamma_{\mu}$, where $\Gamma_{\mu}$ are the connections. From now on we work in the co-rotating frame of reference described by the metric (2.6) and define the connections with respect to this metric. By choosing in these coordinates the appropriate basis of the one-forms we can define the $\gamma$-matrices

$$
\gamma^{t}=\frac{1}{\sqrt{B}} \bar{\gamma}^{t}+\frac{\sqrt{C}}{N^{2}} \tilde{N}^{\varphi} \bar{\gamma}^{\varphi} \quad, \quad \gamma^{\varphi}=\frac{1}{\sqrt{C}} \bar{\gamma}^{\varphi},
$$

where $B$ and $C$ are given by (2.10) and (2.11). The matrices $\bar{\gamma}^{t}$ and $\bar{\gamma}^{\varphi}$ are the standard Dirac $\gamma$-matrices in the corresponding representation,

$$
\left(\bar{\gamma}^{t}\right)^{2}=-1 \quad, \quad\left(\bar{\gamma}^{\varphi}\right)^{2}=1 \quad, \quad\left\{\bar{\gamma}^{\varphi}, \bar{\gamma}^{t}\right\}=0 \quad .
$$

With this definition one has

$$
\left(\gamma^{t}\right)^{2}=g^{t t} \quad, \quad\left(\gamma^{\varphi}\right)^{2}=g^{\varphi \varphi} \quad, \quad\left\{\gamma^{t}, \gamma^{\varphi}\right\}=2 g^{t \varphi} \quad, \quad\left\{\gamma^{i}, \gamma^{j}\right\}=2 g^{i j},
$$


where all $\gamma^{i}$ anticommute with $\gamma^{t}$ and $\gamma^{\varphi}$.

We are interested in single-particle excitations of the spinor field which are the eigenfunctions of the Killing vector $\xi$

$$
\psi(\varphi, t, \mathbf{x})=e^{-i\left(\omega+\Omega_{H} l\right) t} e^{i l \varphi} \psi_{\omega, l}(\mathbf{x})=e^{-i \omega t} e^{i l \tilde{\varphi}} \psi_{\omega, l}(\mathbf{x}) .
$$

By following the method used for the scalar fields we make the Fourier transform

$$
\psi_{\omega}(\varphi, \mathbf{x})=\sum_{l} e^{i l \varphi} \psi_{\omega, l}(\mathbf{x})
$$

The direct computation gives

$$
\gamma^{t} \Gamma_{t}+\gamma^{\varphi} \Gamma_{\varphi}=\frac{1}{4} \gamma^{i} \nabla_{i} \ln (B C)
$$

By using this identity it can be shown that the equation for $\psi_{\omega}$ obtained from (2.42) with the help of (2.43) takes the simple form

$$
\left[(-i \omega) \frac{1}{\sqrt{B}} \bar{\gamma}^{t}+\left(\gamma^{a}\left(\tilde{\nabla}_{a}-i \omega A_{a}-\frac{1}{2} \nabla_{a} \sigma\right)+m\right)\right] \psi_{\omega}=0,
$$

where $a=\{\varphi, i\}$, and $i=1, . ., D-2$. The quantities $A_{a}$ and $\sigma$ are defined by (2.23) and (2.36), respectively. The spin-connections $\tilde{\nabla}_{a}$ are computed with respect to the metric

$$
d l^{2}=C d \varphi^{2}+g_{i j} d x^{i} d x^{j}
$$

Note that the $i$-th component of $\tilde{\nabla}_{a}$ coincides with $i$-th component of the spin-connection $\nabla_{\mu}$ in the physical space-time (2.6). The spectral problem (2.49) can be solved by introducing the fiducial Hamiltonian for spin 1/2 fields

$$
H(\lambda)=i \sqrt{B} \bar{\gamma}^{t}\left[\gamma^{a}\left(\tilde{\nabla}_{a}-i \lambda A_{a}-\frac{1}{2} \nabla_{a} \sigma\right)+m\right] \quad .
$$

The eigen-spinors of $H(\lambda)$,

$$
H(\lambda) \psi_{\omega}^{(\lambda)}=\omega \psi_{\omega}^{(\lambda)}
$$

give the eigen-spinors for Eq. (2.49)

$$
\psi_{\omega}(\varphi, \mathbf{x})=\psi_{\omega}^{(\omega)}(\varphi, \mathbf{x})
$$

In the complete analogy with the case of the scalar fields, one can define fiducial $D$ dimensional spinors

$$
\psi^{(\lambda)}(t, \varphi, \mathbf{x})=\sum_{\omega} e^{-i \omega t} \psi_{\omega}^{(\lambda)}(\varphi, \mathbf{x})
$$

which obey the Dirac equation

$$
\left[\tilde{\gamma}^{\mu}\left(\tilde{\nabla}_{\mu}-i \lambda A_{\mu}\right)+m\right] \psi^{(\lambda)}=0
$$


on fiducial static space-time $\tilde{\mathcal{M}}$ with the metric (2.22) and interact with gauge field (2.23). We see, therefore, that the form of the fiducial background and the gauge field is universal for fields of different spins. This fact may be especially important for supersymmetric models.

The analysis of spinor fields goes along the lines of the work [9]. Firstly, one can see that the spinor Hamiltonian (2.51) is Hermitean with respect to the inner product

$$
\left(\psi_{1}, \psi_{2}\right)=\int d \varphi d^{D-2} \mathbf{x} \sqrt{-g B^{-1}}\left(\psi_{1}(\varphi, \mathbf{x})\right)^{+} \psi_{2}(\varphi, \mathbf{x})
$$

Secondly, for the further convenience, one can go to another representation

$$
\begin{gathered}
e^{-\frac{D-1}{2} \sigma} \bar{H}(\lambda) e^{\frac{D-1}{2} \sigma}=H(\lambda), \\
\bar{H}(\lambda)=i \bar{\gamma}^{t}\left[\bar{\gamma}^{a}\left(\bar{\nabla}_{a}-i \lambda A_{a}\right)+m e^{-\sigma}\right],
\end{gathered}
$$

where the parameter $\sigma$ was introduced in (2.36) and $\gamma$-matrices and spin connections are defined with respect to metric (2.39), which is conformally related to metric (2.50). The operator $\bar{H}(\lambda)$ is Hermitean with respect to the standard inner product

$$
\left(\psi_{1}, \psi_{2}\right)=\int d \varphi d^{D-2} \mathbf{x} \sqrt{\bar{g}}\left(\psi_{1}(\varphi, \mathbf{x})\right)^{+} \psi_{2}(\varphi, \mathbf{x})
$$

The density of energy-levels $d n^{(\lambda)}(\omega) / d \omega$ of the spinor Hamiltonian can be computed with the help of relation (3.3) by using of the heat kernel of the operator

$$
\begin{aligned}
& \bar{H}^{2}(\lambda)=-\bar{g}^{\alpha \beta}\left(\bar{\nabla}_{\alpha}-i \lambda A_{\alpha}\right)\left(\bar{\nabla}_{\beta}-i \lambda A_{\beta}\right)+\bar{V}(\lambda), \\
& \bar{V}(\lambda)=\frac{1}{4} \bar{R}+B\left(m^{2}-m \gamma^{\mu} \nabla_{\mu} \sigma+\frac{i}{2} \lambda \gamma^{\mu} \gamma^{\nu} F_{\mu \nu}\right),
\end{aligned}
$$

where $\bar{R}$ is the curvature of the space (2.39) and $F_{\mu \nu}=A_{\nu, \mu}-A_{\mu, \nu}$ is the Maxwell tensor for the fiducial vector potential.

\section{Properties of $\bar{H}^{2}(\lambda)$ and divergences related to the horizon}

From now on we restrict the discussion by the four-dimensional space-times $(D=4)$. However the analysis can be carried in higher dimensions as well.

Both scalar and spinor single-particle Hamiltonians $\bar{H}(\lambda)$ are defined on the space (2.39). By following the conventions adopted in Ref. [6] we denote this space $\overline{\mathcal{B}}$. In the vicinity of the horizon the geometry of $\overline{\mathcal{B}}$ is simple. If $\rho$ is the proper distance to the horizon then (see Appendix)

$$
N^{2} \simeq \kappa^{2} \rho^{2} \quad, \quad C \simeq g_{\varphi \varphi} \quad, \quad B \simeq N^{2}
$$

where $\kappa$ is the surface gravity of the horizon. Let $D=4$. By using these asymptotics and Eqs. (2.38) and (2.61) one finds that the potential terms at the horizon act as a tachionic 
mass, $\bar{V}=-\kappa^{2}$ for scalars and $\bar{V}=-\frac{3}{2} \kappa^{2}$ for spinors. The presence of the tachionic mass, however, is exactly compensated by the mass gap which appears when a particle moves on the space $\overline{\mathcal{B}}$. Near the horizon $\rho=0$ the metric of $\overline{\mathcal{B}}$ takes the form

$$
d \bar{l}^{2} \simeq \frac{1}{\kappa^{2} \rho^{2}}\left(d \rho^{2}+d \Omega^{2}\right)
$$

where in the limit $\rho \rightarrow 0$ the metric $d \Omega^{2}$ coincides with the metric on the horizon. In this limit the curvature of $\overline{\mathcal{B}}$ is constant, $\bar{R}=-6 \kappa^{2}$ and the space looks as a the hyperbolic (Lobachevsky) manifold. Let us emphasize that these properties are the same as for static space-times [7], [8].

These properties are sufficient to conclude that $\bar{H}^{2}(\lambda)$ has a continuous non-negative spectrum without a mass gap. Thus, the density of eigen-values $d n^{(\lambda)}(\omega) / d \omega$ is divergent and requires a regularization. To calculate this quantity and investigate its divergence we use the method [9] based on the relation

$$
\operatorname{Tr} e^{-\bar{H}^{2}(\lambda) t}=\int_{0}^{\infty} d \omega \frac{d n^{(\lambda)}(\omega)}{d \omega} e^{-\omega^{2} t}
$$

The density $d n^{(\lambda)}(\omega) / d \omega$ can be found from (3.3) in terms of the trace of the operator $\bar{H}^{2}(\lambda)$ by using the inverse Laplace transform. The trace involves the integration over the non-compact space $\overline{\mathcal{B}}$. The volume element of $\overline{\mathcal{B}}$ diverges at small $\rho$ as $\rho^{-3}$ and this is the reason of the divergences of the density of levels.

As was explained in Ref. [9], to study this divergence it is sufficient to restrict oneself only by the asymptotic form of the diagonal element of the heat kernel at small values of the parameter $t$

$$
\left[e^{-\bar{H}^{2}(\lambda) t}\right]_{\operatorname{diag}} \simeq \frac{1}{(4 \pi t)^{3 / 2}}\left(1+\bar{a}_{1}(\lambda) t+\bar{a}_{2}(\lambda) t^{2}+\ldots\right)
$$

At this point one can make an important observation. The gauge potential $A_{\alpha}$ appears in the heat kernel only in the gauge invariant combinations. Moreover, the coefficient $\bar{a}_{1}$ does not depend on $A_{\alpha}$ and it is is the same as in the case $\lambda=0$. The coefficient $\bar{a}_{2}(\lambda)$ includes the Maxwell Lagrangian constructed of $A_{\alpha}$. The latter term vanishes as $\rho^{4}$ and it does not bring the divergence to the trace at small $\rho$. The same happens in the higher order coefficients which vanish at least as fast as $\bar{a}_{2}(\lambda)$.

Thus, we come to the conclusion that in four-dimensional space-time the fiducial gauge field does not change the divergence. If one is interested only in the divergent part of density of levels, the parameter $\lambda$ in the energy operator $H^{2}(\lambda)$ can be put equal to zero. This fact reduces our problem to Eqs. (2.21), (2.55) on the static space-time $\tilde{\mathcal{M}}$ with the gauge field neglected. The divergence of the density of levels can be now computed by using the results of [6], [9] and expressed in terms of the geometrical characteristics of $\tilde{\mathcal{M}}$ near the horizon. 
To put it in a more formal way, in four dimensions the regularized divergent part of the density of levels of a field near a rotating black hole

$$
\left[\frac{d n(\omega \mid \mu)}{d \omega}\right]_{d i v}=\left[\frac{d n^{(\lambda=0)}(\omega \mid \mu)}{d \omega}\right]_{d i v}
$$

where $\mu$ is a regularization parameter. By working, for instance, in the Pauli-Villars regularization and by using the expressions of Refs. [6], [9], one finds

$$
\begin{gathered}
{\left[\frac{d n_{s}^{(0)}(\omega \mid \mu)}{d \omega}\right]_{d i v}=\frac{1}{(4 \pi)^{2} \kappa} \int_{\Sigma}\left[2 b+a\left(\frac{\omega^{2}}{\kappa^{2}} \mathcal{P}+2\left(\frac{1}{6}-\xi\right) R\right)\right],} \\
{\left[\frac{d n_{d}^{(0)}(\omega \mid \mu)}{d \omega}\right]_{d i v}=\frac{r_{d}}{(4 \pi)^{2} \kappa} \int_{\Sigma}\left[2 b+a\left(\frac{\omega^{2}}{\kappa^{2}} \mathcal{P}+\frac{R}{6}+\frac{\mathcal{Q}}{4}\right)\right]}
\end{gathered}
$$

Expressions (3.6) and (3.7) are referred to the scalar and spinor densities of levels, respectively, $r_{d}$ being the dimensionality of the spinor representation. The integrals in these expressions are taken over the bifurcation surface $\Sigma$ of the horizon. As we show in Appendix, the curvatures of the physical space-time $\mathcal{M}$ and the fiducial one $\tilde{\mathcal{M}}$ coincide near $\Sigma$. Thus, the quantity $R$ in (3.6), (3.7) can be identified with the scalar curvature of $\mathcal{M}$, while other quantities can be written in terms of the Riemann and Ricci tensors of $\mathcal{M}$

$$
\mathcal{P}=2 \mathcal{R}-\mathcal{Q} \quad, \quad \mathcal{Q}=P^{\mu \nu} R_{\mu \nu} \quad, \quad \mathcal{R}=P^{\mu \nu} P^{\lambda \rho} R_{\mu \lambda \nu \rho}
$$

where $P^{\mu \nu}=l^{\mu} l^{\nu}-p^{\mu} p^{\nu}$ is a projector onto a two-dimensional surface orthogonal to $\Sigma$, and $p^{\mu}, l^{\mu}$ are two mutually orthogonal normals of $\Sigma\left(l^{2}=-p^{2}=1\right)$. The regularization parameter $\mu$ defines the scale of the Pauli-Villars masses, and at large $\mu$

$$
a \simeq \ln \frac{\mu^{2}}{m^{2}} \quad, \quad b \simeq \mu^{2} \ln \frac{729}{256}-m^{2} \ln \frac{\mu^{2}}{m^{2}}
$$

where $m$ is the mass of the field (see for details [6]).

Note that the form of these equations is completely the same as in the static spacetimes. By using (3.6), (3.7) one can find the divergences of the free energy of the fields and the entropy which just repeat expressions (4.26) and (4.27) of Ref. [6]. For instance, in the Pauli-Villars regularization the divergence of the entropy of the quanta near the horizon is given by the expression

$$
S_{\mathrm{div}}=\frac{\eta}{48 \pi} \int_{\Sigma}\left[b f_{1}+a\left(2 p_{1} \mathcal{P}+p_{2} R+p_{3} \mathcal{Q}\right)\right]
$$

For scalars $\eta=1, f_{1}=1, p_{1}=1 / 60, p_{2}=1 / 6-\xi, p_{3}=0$; for spinors $\eta=-1$, $f_{1}=-r_{d} / 2, p_{1}=-7 r_{d} / 480, p_{2}=r_{d} / 24, p_{3}=-r_{d} / 16$. The entropy is evaluated at the Hawking temperature $\beta_{H}^{-1}=\kappa / 2 \pi$. For scalar fields the same result was recently obtained by the WKB method in [14]-15]. Also in the scalar case one can find the divergent part of the entropy by using the Euclidean formalism (conical singularity method), see [17]. 
Analogous results can be found in the dimensional regularization. It should be noted that the divergences caused by the presence of the horizon can be also regularized by using the infrared type cutoff. In this regularization one just cuts all integrations near the horizon at some proper distance, see for a review [6]. Our results can be used to find explicit expressions for the entropy in this case, however, the discussion of this regularization is beyond the scope of this paper. For rotating black hole space-times this question was studied in Refs. [10]-13.

The fact that divergent part (3.10) of the entropy of quantum fields near a rotating black hole has the same form as for a static black hole has a number of immediate consequences. One of the consequences is that for minimally coupled fields divergence (3.10) is completely removed by the standard renormalization of the gravitational couplings (including the Newton constant) in the bare tree-level part of the black hole entropy. The proof of this statement for static black holes can be found in Refs. [19-22] and it is generalized without changes to rotating black holes. Another application of our results is the problem of black hole entropy in models of induced gravity.

\section{Rotating black holes in induced gravity}

The models of induced gravity [23] 25] were constructed with the purpose to understand the mechanism of the generation the Bekenstein-Hawking entropy of black holes in the situation when the low-energy gravity is induced by quantum effects. It was argued that for a Schwarzschild black hole the ultraheavy fields (constituents) which induce the Einstein gravity in the low-energy limit are microscopic degrees of freedom which are responsible for the Bekenstein-Hawking entropy $S^{B H}$. The important requirement of models [23]-25] is the absence of the leading ultraviolet divergences, which imposes constraints on the parameters of the constituents. By using these constraints one finds the relation between $S^{B H}$ and the entropy $S$ of the constituents propagating near the black hole horizon

$$
S^{B H}=S-Q
$$

The quantity $Q$ is the quantum average of the Noether charge [25, 29] which appears because of non-minimal couplings of the constituents with the curvature. Such couplings are necessary to provide cancellation of the leading ultraviolet divergences in the induced effective action. It is important that the same couplings provide finiteness of the induced Bekenstein-Hawking entropy (4.1): the divergence (3.10) of the entropy $S$ of the constituents is compensated by the divergence of the Noether charge $Q$.

We now have all means to generalize result (4.1) of [23]-25] to Kerr black holes. Consider induced gravity models with spinor and non-minimally coupled scalar constituents only. The constraints on the parameters of the constituents and proof of relation (4.1) for a Schwarzschild black hole are given in [23]. The Kerr black hole is the vacuum solution 
and the geometrical structure of the divergences in the effective action for the Kerr and Schwarzschild backgrounds are identical. The induced effective action for a Schwarzschild solution contains logarithmic divergences of a topological form only. These divergences play no role and can be neglected [23]. We conclude that the same property is true for the action on the Kerr background. In this sense the induced gravity [23]-25] for vacuum static and rotating black hole is ultraviolet finite theory.

Consider now the divergence of the entropy $S$ for a scalar or spinor constituent, see Eq. (3.10). According to Eqs. (A.16), (A.24) of Appendix A,

$$
\mathcal{Q}=0 \quad, \quad \int_{\Sigma} \mathcal{R}=8 \pi
$$

for the Kerr background. Thus,

$$
S_{\text {div }}=\frac{\eta}{48 \pi} b f_{1} \mathcal{A}+C
$$

where $\mathcal{A}=\int_{\Sigma}$ is the area of the black hole horizon and $C$ is a divergent numerical constant (which is not observable and can be neglected). Thus, the entropy of the constituents in the leading order is proportional to the area of the horizon of the Kerr black hole and looks similar to the Bekenstein-Hawking entropy. Equation (4.3) has precisely the same form as the entropy for a Schwarzschild black hole. As far the Noether charge $Q$ is concerned, in the considered models it is determined by the averages of the scalar operators $\left\langle\hat{\phi}^{2}\right\rangle$ on the horizon $\Sigma$. In quantum states where the Green functions are analytical on the horizon

$$
\int_{\Sigma}\left\langle\hat{\phi}^{2}\right\rangle=\frac{1}{16 \pi^{2}} b \mathcal{A}
$$

where the function $b$ is given in Pauli-Villars regularization by (3.9). This equation holds on all vacuum backgrounds in the leading order approximation, and one can conclude that the Noether charges $Q$ for the two black holes have the same form.

These observations show that in induced gravity models Eq. (4.1) does hold for the Bekenstein-Hawking entropy of a Kerr black hole. By using (3.9), (4.3), (4.4) in (4.1) one can check how divergence of $Q$ compensates the divergence of $S$ and one gets a finite expression which coincides with the induced entropy $S^{B H}$. It is a strong support of universality of the statistical-mechanical explanation of the Bekenstein-Hawking entropy in induced gravity.

We complete this Section with remarks concerning the interpretation of the Noether charge $Q$. The origin of subtraction in (4.1) can be explained as follows [25]. The Bekenstein-Hawking entropy of a rotating black hole in induced gravity is related to the spectrum of the black hole mass $M$ and angular momentum $J$ which determine the grand canonical ensemble. On the other hand the statistical-mechanical entropy $S$ is determined by the spectrum of the Hamiltonian $\mathcal{H}_{\xi}$ of the constituents. Operator $\mathcal{H}_{\xi}$ is the generator of canonical transformations of the system along the Killing field $\xi$. In the presence of 
non-minimal couplings these spectra are different and subtraction of $Q$ in (4.1) is required to go from one spectrum to another.

To make this statement more clear consider a small excitation of constituent field having energy $\mathcal{E}$ and angular momentum $\mathcal{J}$ over a vacuum with $\mathcal{E}=\mathcal{J}=0$. Such an excitation results in a change of the black hole mass $M$ and angular momentum $J$

$$
\delta M=T_{H} \delta S^{B H}+\Omega_{H} \delta J+\mathcal{E}-\Omega_{H} \mathcal{J}
$$

see [29]. Strictly speaking, this relation implies definition of $M$ and $J$ at spatial infinity. For this reason, the energy and angular momentum of fields are the integrals over all black hole exterior 29]

$$
\begin{gathered}
\mathcal{E}=\int_{\Sigma_{t}} T^{\mu \nu} t_{\mu} d \Sigma_{\nu}, \\
\mathcal{J}=-\int_{\Sigma_{t}} T^{\mu \nu} \varphi_{\mu} d \Sigma_{\nu},
\end{gathered}
$$

where $T^{\mu \nu}$ is the stress-energy tensor of the fields. $\Sigma_{t}$ is the hypersurface of constant time $t$ and $d \Sigma_{t}$ is the future-directed vector of the volume element of $\Sigma_{t}$. The components $t_{\mu}$, $\varphi_{\mu}$ correspond to the Killing vector fields $\partial_{t}$ and $\partial_{\varphi}$, respectively.

In the induced gravity approach the constituents which contribute to the black hole entropy are assumed to be very heavy and have the mass of the order of the Planckian mass. Since Hawking and the superradiant emissions of such particles are exponentially suppressed, they are practically trapped inside the potential barrier. The latter in many aspects plays the role of the external boundary which is required to define the canonical ensemble.

Thus, the dominant contribution to integrals (4.6), (4.7) comes from the region inside the null 'cylinder' (see discussion in Section 2.1) where one can define the energy of constituents associated to the Killing field $\xi=\partial_{t}+\Omega_{H} \partial_{\varphi}$

$$
\mathcal{E}_{\xi}=\mathcal{E}-\Omega_{H} \mathcal{J}=\int_{\Sigma_{t}} T^{\mu \nu} \xi_{\mu} d \Sigma_{\nu}
$$

After that variational formula (4.5) is represented as

$$
\delta M-\Omega_{H} \delta J=T_{H} \delta S^{B H}+\mathcal{E}_{\xi}
$$

and it looks somewhat similar to the formula for static black holes. Thus, for a black hole with the fixed area the spectrum of $M$ and $J$ is related to the spectrum of energies $\mathcal{E}_{\xi}$ of the constituents near the horizon. The crucial observation is that the energy $\mathcal{E}_{\xi}$ and the Hamiltonian $\mathcal{H}_{\xi}$ of the non-minimally coupled constituents differ by a total derivative which picks up a non-vanishing contribution on the inner boundary of $\Sigma_{t}$, i.e., on the horizon. The boundary term is the Noether charge on $\Sigma$

$$
\mathcal{H}_{\xi}-\mathcal{E}_{\xi}=T_{H} Q
$$


where $T_{H}$ is the Hawking temperature. It is because of Eqs. (4.9), (4.10) we expect that the two entropies, $S^{B H}$ and $S$, are different and related by (4.1). Studying further aspects of the subtraction in (4.1) repeats the analysis of a Schwarzschild black hole and we advise corresponding work [25] for the interested reader.

\section{Concluding remarks}

Our results can be summarized as follows. We developed a formalism of statisticalmechanical computations for a canonical ensemble of fields near the horizon of a rotating black hole. Such a canonical ensemble can be defined when the reference frame co-rotates with the angular velocity of the black hole. We suggested a method how to reduce computations on the stationary background to computations on a fiducial static spacetime in the presence of a fiducial gauge potential. Our method enables one to use the known results for this problem and to get a number of rigorous results for rotating black holes. We believe that the method may be helpful in a number of applications, some of which were discussed in Sections 3 and 4. In particular, it is worth pointing out here the proof of universality of statistical-mechanical origin of the Bekenstein-Hawking entropy of vacuum black holes in the models of induced gravity.

One of the results of our analysis is that the Euclidean formulation of the theory based on the conical-singularity method [17] reproduces correctly the divergence of the entropy (3.10) for stationary space-times. In spite of this fact, the equivalence between the canonical formulation of statistical mechanics and the Euclidean one remains unclear in this case. Unfortunately, one cannot apply the analysis of [9] given for static geometries. The difficulty is related not to the horizon but to the prescription used for the Euclidean theory which implies an analytical continuation of some parameters of the metric. This issue is an interesting problem for further research.

Acknowledgments: The work of V.F. is partially supported by the Natural Sciences

and Engineering Research Council of Canada, and D.F. is supported in part by the RFBR grant N 99-02-18146. 


\section{A Geometry of $\mathcal{M}$ and $\tilde{\mathcal{M}}$ near the horizon}

In this appendix, we consider four-dimensional space-times. Generalization of the results to higher dimensions is straightforward. Consider first a line element $(i, j=2,3)$

$$
d L^{2}=g_{i j} d x^{i} d x^{j}
$$

which enters metrics (2.6) and (2.22) of spaces $\mathcal{M}$ and $\tilde{\mathcal{M}}$, respectively,

$$
\begin{gathered}
d s^{2}=-B d t^{2}+2 g_{\varphi \varphi} \tilde{N}_{\varphi} d t d \tilde{\varphi}+g_{\varphi \varphi} d \tilde{\varphi}^{2}+d L^{2} \\
d \tilde{s}^{2}=-B d t^{2}+C d \tilde{\varphi}^{2}+d L^{2}
\end{gathered}
$$

Starting with an arbitrary surface $\mathcal{S}$ and introducing geodesic coordinates one can always rewrite (A.1) in the form

$$
d L^{2}=d \rho^{2}+v(\rho, x) d x^{2} .
$$

It is convenient to choose the surface $\mathcal{S}$ where $\rho=0$ to coincide with the horizon. For given metric (A.1) it is sometimes difficult to solve the geodesic equations required for the coordinate transformation which results in (A.4). Much easier problem is the reduction of the metric (A.1) to the form

$$
d L^{2}=d \rho^{2}+2 q(\rho, x) d \rho d x+v(\rho, x) d x^{2} .
$$

We shall use this form for further calculations.

It can be shown that for a geometry which is regular at the horizon the following decompositions of the metric coefficients is valid near the horizon (i.e., at small $\rho$ )

$$
\begin{gathered}
B=\kappa^{2} \rho^{2}\left(1+b(x) \rho^{2}+O\left(\rho^{4}\right)\right) \\
g_{\varphi \varphi} \tilde{N}^{\varphi}=\rho^{2} p_{1}(x)+O\left(\rho^{4}\right) \\
g_{\varphi \varphi}=f_{1}(x)+f_{2}(x) \rho^{2}+O\left(\rho^{4}\right) \\
v=v_{1}(x)+v_{2}(x) \rho^{2}+O\left(\rho^{4}\right) \\
q=\rho q_{1}(x)+O\left(\rho^{3}\right) \\
C=f_{1}(x)+\left(f_{2}(x)+\frac{p_{1}^{2}(x)}{\kappa^{2}}\right) \rho^{2}+O\left(\rho^{4}\right)
\end{gathered}
$$

The constant $\kappa$ is the surface gravity of the black hole horizon. Equation (A.11) follows from Eqs. (2.8) and (2.11).

Now, by direct computation, one can express the components of the Riemann and Ricci tensors on the horizon in terms of the coefficients present in (A.6)-(A.11). Let us define on the bifurcation surface $\Sigma$ of the horizon the following quantities

$$
\mathcal{Q}=P^{\mu \nu} R_{\mu \nu} \quad, \quad \mathcal{R}=P^{\mu \nu} P^{\lambda \rho} R_{\mu \lambda \nu \rho} \quad, \quad P^{\mu \nu}=l^{\mu} l^{\nu}-p^{\mu} p^{\nu},
$$

\footnotetext{
${ }^{2}$ These definitions coincide with (3.8).
} 
where $P^{\mu \nu}$ is the projector onto two-dimensional surface orthogonal to $\Sigma$. For the space $\mathcal{M}$

$$
\begin{gathered}
\mathcal{R}=2 R_{\rho t \rho}^{t}=-6 b-2 \frac{q_{1}^{2}}{v_{1}}, \\
\mathcal{Q}=2\left[-3 b-\frac{q_{1}^{2}}{v_{1}}+\left(\frac{q_{1}}{v_{1}}\right)^{\prime}-\frac{v_{2}}{v_{1}}-\frac{1}{f_{1}}\left(f_{2}+\frac{p_{1}^{2}}{\kappa^{2}}\right)+\frac{g_{1}}{2 v_{1}}\left(\frac{v_{1}^{\prime}}{v_{1}}+\frac{f_{1}^{\prime}}{f_{1}}\right)\right],
\end{gathered}
$$

where $f^{\prime} \equiv d f / d x$. Then, by using (A.6), A.9)-(A.11) one verifies that for the space $\tilde{\mathcal{M}}$

$$
\tilde{\mathcal{R}}=\mathcal{R} \quad, \quad \tilde{\mathcal{Q}}=\mathcal{Q}
$$

According to the Gauss-Codacci equations

$$
R=R_{\Sigma}+2 \mathcal{Q}-\mathcal{R}
$$

where $R$ and $R_{\Sigma}$ are scalar curvatures of $\mathcal{M}$ and $\Sigma$, respectively. (Here we took into account that the extrinsic curvatures of $\Sigma$ vanish due to the isometry). The same equation is valid for the scalar curvature $\tilde{R}$ of $\tilde{\mathcal{M}}$ and one concludes that

$$
\tilde{R}=R
$$

Therefore, all the curvatures which characterize the geometry of the physical $\mathcal{M}$ and fiducial $\tilde{\mathcal{M}}$ space-times coincide at the horizon $\Sigma$. As far as other geometrical properties (e.g., derivatives of the curvatures at $\Sigma$ ) are concerned, they can be different in general. This fact, however, is not important when one studies the divergences of the density of energy-levels in four-dimensional theory, Section 1.3.

For the sake of completeness, we give the expressions for the surface invariants for $\mathcal{Q}$ and $\mathcal{R}$ for the Kerr-Newman black hole of mass $M$, charge $Q$, and angular momentum $J=a M$. The metric in Boyer-Lindquist coordinates is

$$
\begin{gathered}
d s^{2}=-\left(1-\frac{2 M r-Q^{2}}{\Sigma}\right) d t^{2}-2 \frac{\left(2 M r-Q^{2}\right) a \sin ^{2} \theta}{\Sigma} d t d \varphi \\
+\frac{\Sigma}{\Delta} d r^{2}+\Sigma d \theta^{2}+\frac{A \sin ^{2} \theta}{\Sigma} d \varphi^{2}, \\
\Delta=r^{2}-2 M r+a^{2}+Q^{2} \quad, \quad \Sigma=r^{2}+a^{2} \cos ^{2} \theta, \\
A=\left(r^{2}+a^{2}\right)^{2}-\Delta a^{2} \sin ^{2} \theta .
\end{gathered}
$$

The horizon is defined by the equation $\Delta=0$ and is located at

$$
r=r_{+}=M+\sqrt{M^{2}-Q^{2}-a^{2}}
$$

The surface gravity $\kappa$ and the angular velocity $\Omega_{H}$ for the Kerr-Newman black hole are

$$
\kappa=\frac{r_{+}-M}{r_{+}^{2}+a^{2}} \quad, \quad \Omega_{H}=\frac{a}{r_{+}^{2}+a^{2}} .
$$


The Kerr-Newmann metric (A.18) can be brought to form (A.2) when one goes to the corotating coordinate frame by the substitution $\varphi=\tilde{\varphi}+\Omega_{H} t$. The coordinates $\theta$ and $x$ in (A.2) and (A.18) coincide, the coordinate $\rho$ is determined as

$$
\rho=\int_{r_{+}}^{r} d r \sqrt{g_{r r}(r, \theta)} .
$$

By using Eqs. (A.18)-(A.22) one can find the coefficients $b, p_{1}, v_{i}, f_{i}$, and $q_{i}$. After some simple algebra one finds from Eqs. (A.13) and (A.14)

$$
\begin{gathered}
\mathcal{Q}=-\frac{2 Q^{2}}{\Sigma_{+}^{2}}, \\
\mathcal{R}=\frac{2}{\Sigma_{+}^{3}}\left(4 r_{+}^{2}\left(2 M r_{+}-Q^{2}\right)+\Sigma_{+}\left(Q^{2}-6 M r_{+}\right)\right),
\end{gathered}
$$

where $\Sigma_{+}$is the value of $\Sigma$ at $r=r_{+}$. The scalar curvature $R$ of the Kerr-Newmann solution vanishes everywhere. 


\section{References}

[1] W.H. Zurek and K.S. Thorne, Phys. Rev. Lett. 542171 (1985).

[2] G.'t Hooft, Nucl. Phys. 256, 727 (1985).

[3] L. Bombelli, R.K. Koul, J. Lee, and R. Sorkin, Phys. Rev. D34, 373 (1986).

[4] M. Strednicki, Phys. Rev. Lett. 71 (1993) 666.

[5] V. Frolov and I. Novikov, Phys. Rev. D48, 4545 (1993).

[6] V.P. Frolov and D.V. Fursaev, Class. Quantum Grav. 15, 2041 (1998).

[7] G. Cognola, L. Vanzo and S. Zerbini, Class. Quantum Grav. 12 (1995) 1927.

[8] A.A. Bytsenko, G. Cognola, and S. Zerbini, Nucl. Phys. B458 (1996) 267.

[9] D.V. Fursaev, Nucl. Phys. B524, 447 (1998).

[10] M.H. Lee and J.K. Kim, Phys. Rev. D54, 3904 (1996).

[11] S.W. Kim, W.T. Kim, Y.J. Park, and H. Shin, Phys. Lett. B392, 311 (1997).

[12] J. Ho, W.T. Kim, Y.J. Park, and H. Shin, Class. Quantum Grav. 14, 2617 (1997).

[13] J. Ho and G. Kang, Phys. Lett. B445, 27 (1998).

[14] J. Jing and M.L. Yan, Quantum Entropy of the General Non-extreme Stationary Axisymmetric Black Hole due to Minimally Coupled Quantum Scalar Field, E-print Archive: gr-qc/9904001.

[15] J. Jing and M.L. Yan, Entropies of the general non-extreme stationary axisymmetric black hole: statistical mechanics and thermodynamics, E-print Archive: grqc/9907011.

[16] G. Cognola, Phys. Rev. D57, 6292 (1998).

[17] R.B. Mann and S. Solodukhin, Phys. Rev. D54, 3932 (1996)

[18] R.B. Mann and S. Solodukhin, Phys. Rev. D55, 3622 (1997).

[19] L. Susskind and J. Uglum, Phys. Rev. D50, 2700 (1994).

[20] C. Callan and F. Wilczek, Phys. Lett. B333, 55 (1994).

[21] D.V. Fursaev and S.N. Solodukhin, Phys. Lett. B365, 51 (1996).

[22] J.-G. Demers, R. Lafrance, and R.C. Myers, Phys. Rev. D52, 2245 (1995). 
[23] V.P. Frolov, D.V. Fursaev, and A.I. Zelnikov, Nucl. Phys. B486, 339 (1997).

[24] V.P. Frolov and D.V. Fursaev, Phys. Rev. D58, 124009 (1998).

[25] V.P. Frolov and D.V. Fursaev, Phys. Rev. D56 2212 (1997).

[26] D. Kramer, H. Stephani, M. Maccallum, and E. Herlt, Exact Solutions of the Einstein Field Equations, Deutscher Verlag Wiss., Berlin (1980).

[27] S. W. Hawking and G. F. Ellis. The Large-Scale Structure of Spacetime, Cambridge Univ. Press (1973).

[28] W. Israel, Phys. Lett. 57A, 107 (1976).

[29] D.V. Fursaev, Phys. Rev. D59, 064020 (1999). 